\title{
The Optimal Allocation of Prizes in Contests
}

\author{
Benny Moldovanu and Aner Sela*
}

July 14, 1999

\begin{abstract}
We study a contest with multiple (not necessarily equal) prizes. Contestants have private information about an ability parameter that affects their costs of bidding. The contestant with the highest bid wins the first prize, the contestant with the second-highest bid wins the second prize, and so on until all the prizes are allocated. All contestants incur their respective costs of bidding. The contest's designer maximizes the expected sum of bids. Our main results are: 1) We display bidding equlibria for any number of contestants having linear, convex or concave cost functions, and for any distribution of abilities. 2) If the cost functions are linear or concave, then, no matter what the distribution of abilities is, it is optimal for the designer to allocate the entire prize sum to a single "first" prize. 3 ) We give a necessary and sufficient conditions ensuring that several prizes are optimal if contestants have a convex cost function.
\end{abstract}

\section{Introduction}

In 1902 Francis Galton posed the following problem:

"A certain sum, say $£ 100$, is available for two prizes to be awarded at a forthcoming competition; the larger one for the first of the competitors, the smaller one for the second. How should the $£ 100$ be most suitably divided between the two ? What ratio should a first prize bear to that of a second one ? Does it depend on the number of competitors, and if so, why ?" Galton (Biometrika, Vol.1, 1902)

${ }^{*}$ We wish to thank Karsten Fieseler, Martin Hellwig, Roman Inderst, Philippe Jehiel, Holger Müller, Georg Nöldeke and Jan Vleugels for helpful comments. Both authors are grateful for financial support from the SFB 504 at the University of Mannheim. Moldovanu: Economics, University of Mannheim, Seminargebaeude A5, 68131 Mannheim. mold@pool.uni-mannheim.de. Sela: Economics, Ben-Gurion Universiy, P.O.B. 653, Beer-Sheva 84105, Israel. 
In his article Galton proposes a ratio of 3 to 1 to the above question. Since Galton does not explicitly state what is the contest designer's goal, his answer is somewhat arbitrary. Nevertheless, his work is important for it pioneered both the scientific literature on contests and the use of order statistics (The ratio 3 to 1 appears as the ratio of expected differences involving the first three order statistics for a large number of contestants whose abilities are normally distributed.)

Many economic, social and biological situations are contests where agents spend resources in order to increase to probability of winning a prize. Several well known examples are: rent-seeking and lobbying inside organizations; R\&D rivalry; sport competitions; arms races and other economic or biological fights and "wars of attrition"; job promotion in labor markets; political campaigns; artistic competitions such as piano or architectural contests.

Given the wealth of examples, it is not surprising that the economic literature on contests is very large. Most of the literature has focused on the case of one prize. Models with complete information about the value of the prize include: Tullock (1980), Varian (1980), Rosen (1986), Hillman and Samet (1987), Baye et al (1996). The last paper offers a complete characterization of equilibrium behavior. Models with incomplete information about the prize's value to different contestants include Weber (1985), Hillman and Riley (1989), Amann and Leininger (1996), and Krishna and Morgan (1997).

Clark and Riis (1998) study contests with multiple identical prizes under complete information and focus on simultaneous versus sequential designs. Barut and Kovenock (1998) and Glazer and Hassin (1988) allow for non-identical prizes in a complete information model. The latter paper also includes a result for a model with incomplete information (see below).

The use of contests in order to extract effort under "moral hazard" conditions has been emphasized by, among others, Lazear and Rosen (1981), Green and Stokey (1981), and Nalebuff and Stiglitz (1983). A common assumption in these papers is that (observed) output is a stochastic function of the unobservable effort. All agents have the same (known) ability. Lazear and Rosen derive the optimal prize structure in a contest with two workers and two prizes (so that, in fact, only the difference in prizes matters) and compare it to optimal piece rates. They also briefly discuss a model with heterogenous workers and the efficiency properties of having contests where such workers mix.

In this paper we address Galton's problem in the following framework: Several agents engage in a contest where multiple prizes with known and common values are awarded. Each contestant $i$ submits a bid (or undertakes an observable "effort"). The contestant with the highest bid wins the first prize, the contestant with the second-highest bid wins the second prize, and so on until all the prizes are allocated. All contestants (including those that did not win any prize) incur 
a cost that is a strictly increasing function of their bid. This function is common knowledge. We differentiate among the cases where the cost function is, respectively, linear, concave or convex in effort. The cost function of contestant $i$ also depends on a parameter (say "ability") that is private information to that player. The main assumption we make is one of separability between ability and bid in the cost function. Abilities are drawn independently of each other according to an arbitrary distribution function which is common knowledge. We assume that each contestant chooses his bid in order to maximize expected utility (given the other competitors' bids and given the values of the different prizes).

The goal of the contest designer is to maximize the total expected effort (i.e, the expected sum of the bids) at the contest ${ }^{1}$. This fits well in the tournament literature. Good examples are sport competitions, promotions in organizations, architectural contests and the grading of exams.

The designer can determine the number of prizes (i.e., the number of prizes having positive value) and the distribution of the fixed total prize sum among the different prizes.

It is worth to note here the theoretical distinction between having one prize (or several equal prizes) and having several unequal prizes. If there is only one prize, or if there are several equal prizes, each contestant perceives two payoff-relevant alternatives: I win a prize, or I win nothing. Hence, bids will be determined by one variable - the difference in expected payoff between those two alternatives. (Note that the same logic applies if there are two unequal prizes but only two contestants). In contrast, if there are at least two unequal prizes and at least three contestants, each contestant perceives at least three payoff relevant alternatives (I win the first prize, I win the second prize,..., I win nothing). Bids will be determined in a more complex way by several variables - the differences in expected payoff among the various alternatives ${ }^{2}$.

We display bidding equilibria for any number of prizes and contestants, for any distribution of abilities, and for linear, concave or convex cost functions. In order to have a less technical exposition, we focus however on the designer's problem in the case where she can award two (potentially unequal) prizes, and where there are at least three contestants. As explained above, this case already displays the main ingredient for complexity in bidding. Moreover, it will become clear that none of our qualitative results changes if we allow for more than two prizes.

\footnotetext{
${ }^{1}$ In some models, the designer has other goals. For example, in a lobying model the contest designer might not be the beneficiary of the "wasteful" lobying activities, and she might wish to minimize them. Our analysis can be easily extended to other goal functions since we explicitly display bidding equilibria.

${ }^{2}$ The complexity resembles the one appearing in multi-object auctions.
} 
Our answers to Galton's questions are as follows: 1) We show that for any number of contestants having linear or concave cost functions, and for any distribution of abilities, it is optimal for the designer to allocate the entire prize sum to a single "first" prize. 2) We give a necessary and sufficient conditions ensuring that (at least) two prizes are optimal if the contestants have convex cost functions. Depending on the parameters, the optimal prize structure may involve then several equal prizes or different prizes whose ratio can be easily computed.

Several related results appear in Barut and Kovenock (1998) and Glazer and Hassin (1988). Barut and Kovenock study a multi-prize contest where players have linear cost functions and have the same ability. In this symmetric, complete information environment, they show that the optimal prize structure allows any combination of $K-1$ prizes, where $K$ is the number of contestants. (In particular, allocating the entire prize sum to a unique first prize is optimal.) Our result for linear cost functions (that allows for private information, asymmetries among contestants and is independent of the number of contestants) suggests that the only robust optimal prize structure is the one involving a unique first prize.

Besides studying the symmetric equilibria of a complete information model as above, Glazer and Hassin (1988) also propose an incomplete information model that is more general than ours since it allows for both cost functions that are not necessarily separable in ability and bid and for a concave prize-valuation function. But they are not able to compute equilibria, and their indirect results (based on several implicit and unproven assumptions) only deal with the case of a separable and linear cost function, a linear prize-valuation function and a uniform ability distribution such that the lowest ability type has an infinite cost of bidding. With these assumptions, they show that a unique first prize is optimal.

We now want to describe the intuition behind our main results. The equilibrium bid function for contestants with linear cost functions is a function of the values of the different prizes and of the ability of that contestant. If there are $p$ prizes, this function involves the distribution of the first $p$ order-statistics. Moreover, the equilibrium bid is increasing in ability. A nice property of the differential equation arising from the first-order condition of the contestants' maximization problem allows us to easily compute the equilibrium bid for contestants with general (i.e., concave or convex) cost functions: this is simply equal to the inverse of the cost function applied to the equilibrium bid for linear cost functions.

To simplify the exposition, we assume for the following descriptive details that the contestants have linear cost functions.

The marginal effect of the first prize on the equilibrium bid function is positive for all possible abilities (or "types"). Since a player with higher ability has a higher chance to win the first prize, the marginal effect of the first price is an increasing function of ability. 
The marginal effect of the second-prize is ambiguous. Consider, for example, a player with the highest possible ability. If such a player is present at the contest, he is sure to win the first prize. Subtracting a penny from the first prize and adding it to the second prize lowers the expected utility of such a player (since he never gets the second prize), and he will consequently bid less. Similarly, the marginal effect of the second prize on the equilibrium bid function is negative for players with high enough abilities (and hence this effect is lower that the marginal effect of the first prize for such types). The marginal effect of the second price is, however, positive for middle and low ability players. Moreover, for contestants with abilities below a certain threshold, the (positive) marginal effect of the second prize is higher than the marginal effect of the first prize, since these types of player have a higher chance to get the second-prize.

Consider now the contest designer: She has to allocate a fixed prize sum among the two prizes, and she wants to maximize the average (i.e., expected) bid of each contestant. The relevant variable for the designer is then the average difference between the marginal effects of the second prize and the first prize, respectively. This average difference is precisely the marginal effect of the second prize on the designer's revenue (i.e., the change in the designer's revenue if she subtracts a penny from the value of the first prize and adds it to the value of the secondprize). If the average marginal effect of the first prize is higher than the average marginal effect of the second-prize then the average difference is negative, the designer's goal function is a decreasing function of the value of the second-prize, and the designer should award only a unique (first) prize - this turns out to be the case for contestants with linear cost functions. To obtain the marginal effect of the second prize on the designer's revenue for contestants with concave cost functions, one multiplies the above difference by a function that is increasing in ability. Hence the terms where the first-prize is dominant (corresponding to high ability types) get magnified in relation to the terms where the second prize is dominant (low ability types). Given the result for the linear cost functions, the designer's revenue is also a decreasing function of the value of the second-prize, and the designer should award only a unique (first) prize. The opposite happens for convex cost functions: the terms where the second prize is dominant get magnified, and two prizes may be optimal (since, by definition, the designer cannot award only a second prize). In such cases, the optimal allocation of the prize sum among the two prizes depends on the number of contestants, the distribution of abilities, and the effort cost function.

The paper is organized as follows: In Section 2 we present the contest model with multiple prizes and private information about a parameter (e.g. ability) entering cost functions. In Section 3 we focus on linear cost functions. We derive the equilibrium bid functions and we formulate the contest designer's problem . 
We then prove several properties that characterize the marginal effects of the firstand the second-prize on each type of contestant . Finally, we use these properties to prove that it is always optimal to award a single prize. In Section 4 we use the result obtained above in order to study the optimal prize structure for contestants with concave and convex cost functions. Finally, we illustrate the (non-trivial) optimal prize structure in an example with convex cost functions. In Section 5 we gather several concluding comments.

\section{The Model}

Consider a contest where $p$ prizes are awarded. The value of the $j-t h$ prize is $V_{j}$, where $V_{1} \geq V_{2} \geq \ldots \geq V_{p}$. The values of the prizes are common knowledge. We assume that $\sum_{i=1}^{p} V_{i}=1$ - this is just a normalization.

The set of contestants is $K=\{1,2, \ldots, k\}$. Without loss of generality we can assume that $k \geq p$ (i.e., there are at least as many contestants as there are prizes).

At the contest each player $i$ makes a bid $x_{i}$. Bids are submitted simultaneously. A bid $x_{i}$ causes a disutility (or cost) denoted by $c_{i} \gamma\left(x_{i}\right)$, where $\gamma: R_{+} \rightarrow R_{+}$ is a strictly increasing function with $\gamma(0)=0$, and where $c_{i}>0$ is an ability parameter $^{3}$. Note that a low $c_{i}$ means that $i$ has a high ability (i.e., lower cost) and vice-versa.

The ability (or type) of contestant $i$ is private information to $i$. Abilities are drawn independently of each other from an interval $[m, 1]$ according to the distribution function $F(\cdot)$, which is common knowledge. We assume that $F(\cdot)$ has a continuous density $F^{\prime}(\cdot)>0$. In order to avoid infinite bids caused by zero costs, we assume that $m$, the type with highest possible ability, is strictly positive ${ }^{4}$.

The contestant with the highest bid wins the first prize $V_{1}$. The contestant with the second highest bid wins the second prize $V_{2}$, and so on until all the prizes are allocated ${ }^{5}$. That is, the payoff of contestant $i$ who has ability $c_{i}$, and submits a bid $x_{i}$ is either $V_{j}-c_{i} \gamma\left(x_{i}\right)$ if $i$ wins prize $j$, or $-c_{i} \gamma\left(x_{i}\right)$ if $i$ does not win a prize.

Each contestant $i$ chooses his bid in order to maximize expected utility (given the other competitors' bids and the values of the different prizes.)

The contest designer determines the number of prizes having positive value and the distribution of the total prize sum among the different prizes in order to

\footnotetext{
${ }^{3}$ The treatment of the case in which $i^{\prime} s$ cost function is given by $\delta\left(c_{i}\right) \gamma\left(x_{i}\right)$, where $\delta(\cdot)$ is strictly monotone increasing, is completely analogous. The main assumption here is the separability of ability and bid.

${ }^{4}$ The case where $m=0$ can be treated as well, but requires slightly different methods.

The choice of the interval $[m, 1]$ is a normalization.

${ }^{5}$ If $h>1$ bids tie for a prize, each respective bidder gets the prize with probability $\frac{1}{h}$.
} 
maximize the expected value of the sum of the bids $\sum_{i=1}^{k} x_{i}$ (given the contestants' equilibrium bid functions).

\section{Linear Cost Functions}

In this Section we assume that the cost functions are linear, i.e., $\gamma(x)=x$. As we shall see below, both the equilibrium bid functions and the optimal prize structure in this case will be very important for the derivations in the other cases.

The next Proposition displays the equilibrium bid when there are two prizes. As mentioned in the introduction, if there are only two contestants, the situation is isomorphic to the one where there is a unique prize whose value is equal to the difference between the two prizes. Hence, it is trivially true that awarding a unique prize is optimal for the contest's designer. Therefore, we assume below that the number of contestants is at least three, i.e., $k \geq 3$. In the Appendix we also provide the general formula for the bid functions with $p>2$ prizes.

Proposition 3.1. In a symmetric equilibrium, the bid function of each contestant is given by $b(c)=A(c) V_{1}+B(c) V_{2}$ where:

$$
\begin{gathered}
A(c)=(k-1) \int_{c}^{1} \frac{1}{a}(1-F(a))^{k-2} F^{\prime}(a) d a \\
B(c)=(k-1) \int_{c}^{1} \frac{1}{a}(1-F(a))^{k-3}\left[((k-1) F(a)-1] F^{\prime}(a) d a\right.
\end{gathered}
$$

Proof. See Appendix

\subsection{The Designer's Problem}

Let $V_{2}=\alpha$ and $V_{1}=1-\alpha$, where $0 \leq \alpha \leq \frac{1}{2}$ (since the second prize must be smaller than the first). By Proposition 3.1, each contestant's equilibrium bid function is given by

$$
b(c)=(1-\alpha) A(c)+\alpha B(c)=A(c)+\alpha(B(c)-A(c)) .
$$

The average bid of each contestant is given by

$$
\int_{m}^{1}(A(c)+\alpha(B(c)-A(c))) F^{\prime}(c) d c .
$$

Since there are $k$ contestants, the seller's problem is:

$$
\max _{0 \leq \alpha \leq \frac{1}{2}} k \int_{m}^{1}(A(c)+\alpha(B(c)-A(c))) F^{\prime}(c) d c
$$


The above problem is equivalent to:

$$
\left.\max _{0 \leq \alpha \leq \frac{1}{2}} \alpha \int_{m}^{1}(B(c)-A(c))\right) F^{\prime}(c) d c
$$

The solution to Problem 3.3 is extremely simple: if the integral is positive, then the optimal $\alpha$ is $\frac{1}{2}$ (i.e., award two equal prizes). Otherwise, the optimal $\alpha$ is zero (i.e., award a unique prize).

The next Proposition shows that the integral appearing in Problem 3.3 is negative. We also list several other properties of the marginal effects, $A(c)$ and $B(c)$, that are used in the proof and in other parts of the paper.

Proposition 3.2. Let $b(c)=A(c) V_{1}+B(c) V_{2}$ be the symmetric equilibrium bid function for contestants having linear cost functions. Then the following properties hold:

1. $A(1)=B(1)=0$

2. $\forall c \in[m, 1), A(c)>0$, and $A^{\prime}(c)<0$

3. Let $c^{*}$ be such that $F\left(c^{*}\right)=\frac{1}{k-1}$. Then $B^{\prime}\left(c^{*}\right)=0, B^{\prime}(c)>0$ for all $c \in\left[m, c^{*}\right)$, and $B^{\prime}(c)<0$ for all $c \in\left(c^{*}, 1\right]$

4. $\left|B^{\prime}(c)\right|>\left|A^{\prime}(c)\right|$ for $c$ in a neighborhood of 1 .

5. $B(m)<0$

6. For any $k>2$, there exists a unique point $c^{* *} \neq 1$ such that $A\left(c^{* *}\right)=B\left(c^{* *}\right)$.

7. $\int_{m}^{1}(B(c)-A(c)) F^{\prime}(c) d c<0$

Proof. See Appendix.

Proposition 3.2-7 shows that the solution to Problem 3.3 must be $\alpha=0^{6}$. Hence we have obtained:

Proposition 3.3. For any number of contestants with linear cost functions, and for any distribution of abilities in the population, it is optimal to allocate the entire prize sum to a single first prize.

\footnotetext{
${ }^{6}$ As noted in the introduction, we can easily deal with the case where the designer wants,say, to minimize the the expected sum of bids. In that case it is obvious by the above analysis that two equal prizes $\left(\alpha=\frac{1}{2}\right)$ are optimal .
} 
The result above holds even if, a-priori, the seller is allowed to award more than two prizes. Indeed, assume that the optimal prize structure is $\left(V_{1}^{*}, V_{2}^{*}, \ldots, V_{p}^{*}\right)$. Let $\beta=1-\sum_{j=3}^{p} V_{j}^{*}$. The marginal effects of the first and second prizes on the equilibrium bids do not depend on $\beta$ (this is similar to the fact that the marginal effect of the first prize does not depend on the value of the second-prize and vice versa - see the general equilibrium bid formula in the Appendix). For any fixed $\beta$, Proposition 3.3 shows that $V_{2}^{*}=0$. Since $V_{j}^{*} \leq V_{2}^{*}$ for all $j>2$, we obtain that $V_{1}^{*}=1$ and that $V_{j}^{*}=0$ for all $j \geq 2$.

The following example illustrates the above results. In particular, we give explicit formulas for the equilibrium bid functions for the case where the distribution of abilities is uniform.

Example 3.4. Assume that $F(c)=\frac{1}{1-m} c-\frac{m}{1-m}$, i.e., abilities are uniformly distributed on the interval $[m, 1]$. We obtain that:

$$
\begin{aligned}
A(c)= & \left(\frac{1}{1-m}\right)^{k-1}(1-k)\left(\sum_{s=1}^{k-2} \frac{(1-c)^{s}}{s}+\ln c\right) \\
B(c)= & \left(\frac{1}{1-m}\right)^{k-1}(k-1)\left[\sum_{s=1}^{k-2} \frac{(1-c)^{s}}{s}+\ln c\right. \\
& \left.+(1-c)^{k-2}+m(k-2)\left(\sum_{s=1}^{k-3} \frac{(1-c)^{s}}{s}+\ln c\right)\right]
\end{aligned}
$$

Assume now that $k=3, m=\frac{1}{2}$ and $F(a)=2 a-1$ (i.e., uniform distribution on the interval $\left.\left[\frac{1}{2}, 1\right]\right)$.

The formulas above yield:

$$
\begin{gathered}
A(c)=-8+8 c-8 \ln c . \\
B(c)=16-16 c+12 \ln c \\
\int_{m}^{1}(B(c)-A(c)) F^{\prime}(c) d c=2 \int_{\frac{1}{2}}^{1}(24-24 c+20 \ln c) d c \\
=-14+20 \ln 2=-0.137
\end{gathered}
$$




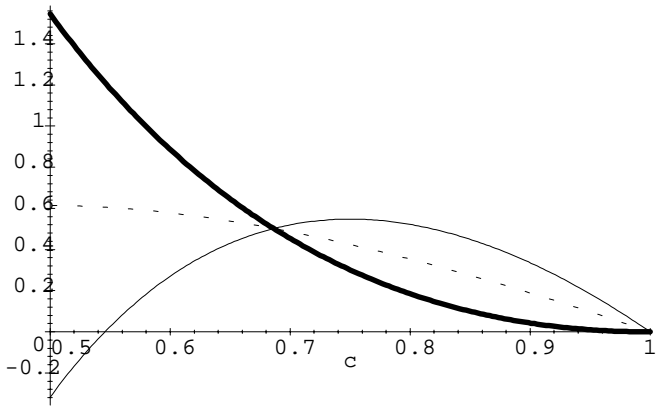

$A(c)$ - thick line ; $B(c)$ - thin line; $\frac{1}{2}(A(c)+B(c))$ - dotted line

Note that the curve $A(c)$ also describes the equilibrium bid when there is a unique price $V_{1}=1$. For comparison, we have also plotted the resulting equilibrium bid function when there are two equal prizes, $V_{1}=V_{2}=\frac{1}{2}$.

\section{Concave and Convex Cost Functions}

Assume now that bidder $i$ with ability $c$ has a cost function given by $c \gamma(\cdot)$ such that $\gamma(0)=0, \gamma^{\prime}(\cdot)>0$. Let $g(\cdot)=\gamma^{-1}(\cdot)$, and observe that $g^{\prime}(\cdot)>0$.

Proposition 4.1. In a symmetric equilibrium, the bid function of each contestant is given by

$$
b(c)=g\left[A(c) V_{1}+B(c) V_{2}\right]
$$

where $A(c)$ and $B(c)$ are defined by equations 3.1 and 3.2, respectively.

Proof. See Appendix.

\subsection{The Designer's Problem}

Let $V_{1}=1-\alpha$, and $V_{2}=\alpha$, where $0 \leq \alpha \leq \frac{1}{2}$. Analogous to the case of linear cost functions, the designer's problem is given by

$$
\max _{0 \leq \alpha \leq \frac{1}{2}} k \int_{m}^{1} g[A(c)+\alpha(B(c)-A(c))] F^{\prime}(c) d c
$$

The designer's revenue as a function of the value of the second prize is:

$$
R(\alpha)=k \int_{m}^{1} g[A(c)+\alpha(B(c)-A(c))] F^{\prime}(c) d c
$$


Note that

$$
R^{\prime}(\alpha)=k \int_{m}^{1}(B(c)-A(c)) g^{\prime}[A(c)+\alpha(B(c)-A(c))] F^{\prime}(c) d c
$$

and that:

$$
R^{\prime \prime}(\alpha)=k \int_{m}^{1}(B(c)-A(c))^{2} g^{\prime \prime}[A(c)+\alpha(B(c)-A(c))] F^{\prime}(c) d c
$$

Observe also that

$$
\begin{aligned}
\frac{d g^{\prime}[A(c)+\alpha(B(c)-A(c))]}{d c}= & g^{\prime \prime}[A(c)+\alpha(B(c)-A(c))] \\
& \cdot\left[(1-\alpha) A^{\prime}(c)+\alpha B^{\prime}(c)\right]
\end{aligned}
$$

and that

$$
(1-\alpha) A^{\prime}(c)+\alpha B^{\prime}(c)<0
$$

since this last term is the derivative of the equilibrium bid function for contestants having linear cost functions. We can now prove the following:

Proposition 4.2. For any number of contestants with concave cost functions, and for any distribution of abilities in the population, it is optimal to allocate the entire prize sum to a single first prize.

Proof. The cost function of contestant $i$ with ability $c, c \gamma(\cdot)$, has the additional feature that $\gamma^{\prime \prime}(\cdot) \leq 0$. Hence $g^{\prime \prime}(\cdot)=\left(\gamma^{-1}(\cdot)\right)^{\prime \prime} \geq 0$. By equations 4.4 and 4.5 we obtain that the positive function $g^{\prime}[A(c)+\alpha(B(c)-A(c))]$ is decreasing in $c$. This means that in the integral defining $R^{\prime}(\alpha)$, all negative terms $B(c)-A(c)$ (corresponding to $c \in\left[m, c^{* *}\right)$ ) are multiplied by relatively high values of $g^{\prime}(\cdot)$, while all positive terms $B(c)-A(c)$ (corresponding to $c \in\left(c^{* *}, 1\right)$ ) are multiplied by relatively lower values. By Proposition 3.2-7, we obtain:

$$
R^{\prime}(\alpha)=k \int_{m}^{1} g^{\prime}[A(c)+\alpha(B(c)-A(c))](B(c)-A(c)) F^{\prime}(c) d c<0
$$

Hence, the designer's payoff function has a maximum at $\alpha=0$, and a single prize is optimal.

Proposition 4.3. Consider a contest where contestants have convex cost functions. A necessary and sufficient condition for the optimality of two prizes is given by

$$
\int_{m}^{1}(B(c)-A(c)) g^{\prime}(A(c)) F^{\prime}(c) d c>0
$$


If condition 4.7 is satisfied ${ }^{7}$ then it is either optimal to award two prizes $V_{1}=1-\alpha^{*}$ and $V_{2}=\alpha^{*}$, where $\alpha^{*}>0$ is determined by the equation $R^{\prime}\left(\alpha^{*}\right)=0$, or to award two equal prizes, $V_{1}=V_{2}=\frac{1}{2}$.

Proof. The cost function of contestant $i$ with ability $c, c \gamma(\cdot)$, has the additional feature that $\gamma^{\prime \prime}(\cdot) \geq 0$. Hence $g^{\prime \prime}(\cdot)=\left(\gamma^{-1}(\cdot)\right)^{\prime \prime} \leq 0$. By equations 4.4 and 4.5 we obtain that the positive function $g^{\prime}[A(c)+\alpha(B(c)-A(c))]$ is increasing in $c$. This means that in the integral defining $R^{\prime}(\alpha)$ all negative terms of the form $B(c)-A(c)$ (corresponding to $c \in\left[m, c^{* *}\right)$ ) are multiplied by relatively low values of $g^{\prime}(\cdot)$, while all positive terms $B(c)-A(c)$ (corresponding to $c \in\left(c^{* *}, 1\right)$ ) are multiplied by higher values. Moreover, for all $\alpha \in\left[0, \frac{1}{2}\right]$ we have

$$
R^{\prime \prime}(\alpha)=k \int_{m}^{1}(B(c)-A(c))^{2} g^{\prime \prime}[A(c)+\alpha(B(c)-A(c))] F^{\prime}(c) d c \leq 0
$$

If condition 4.7 is satisfied then we have:

$$
R^{\prime}(0)=k \int_{m}^{1}\left((B(c)-A(c)) g^{\prime}(A(c)) F^{\prime}(c) d c>0\right.
$$

Hence, the revenue function $R(\alpha)$ cannot have a maximum at $\alpha=0$. It either has a maximum at $\alpha^{*}$ such that $R^{\prime}\left(\alpha^{*}\right)=0$ or at $\alpha=\frac{1}{2}$.

For the converse, assume that two prizes are optimal. This means that $\alpha=0$ is not a maximum of $R(\alpha)$. If condition 4.7 is not satisfied we obtain $R^{\prime}(0) \leq 0$. Together with $R^{\prime \prime}(\alpha) \leq 0$ for all $\alpha \in\left[0, \frac{1}{2}\right]$ we obtain a contradiction.

Example 4.4. Let $k=3, m=\frac{1}{2}$ and $F(a)=2 a-1$ (i.e., uniform distribution on the interval $\left.\left[\frac{1}{2}, 1\right]\right)$. Let the cost function be $c \gamma(x)=c x^{2}$. We have $\gamma^{-1}(x)=$ $g(x)=x^{\frac{1}{2}}$ and $g^{\prime}(x)=\frac{1}{2} x^{-\frac{1}{2}}$. By the results in Example 3.4, we obtain:

$$
\begin{aligned}
A(c) & =-8+8 c-8 \ln c \\
B(c) & =16-16 c+12 \ln c \\
B(c)-A(c) & =24-24 c+20 \ln c \\
g^{\prime}(A(c)) & =\frac{1}{2}(-8+8 c-8 \ln c)^{-\frac{1}{2}} \\
\int_{\frac{1}{2}}^{1}(B(c)-A(c)) g^{\prime}(A(c)) F^{\prime}(c) & =\sqrt{2} \int_{\frac{1}{2}}^{1} \frac{6-6 c+5 \ln c}{\sqrt{(-1+c-\ln c)}} d c=0.19
\end{aligned}
$$

\footnotetext{
${ }^{7}$ Note that the condition involves only primitives of the model: the distribution function, the cost function, and the number of contestants.
} 


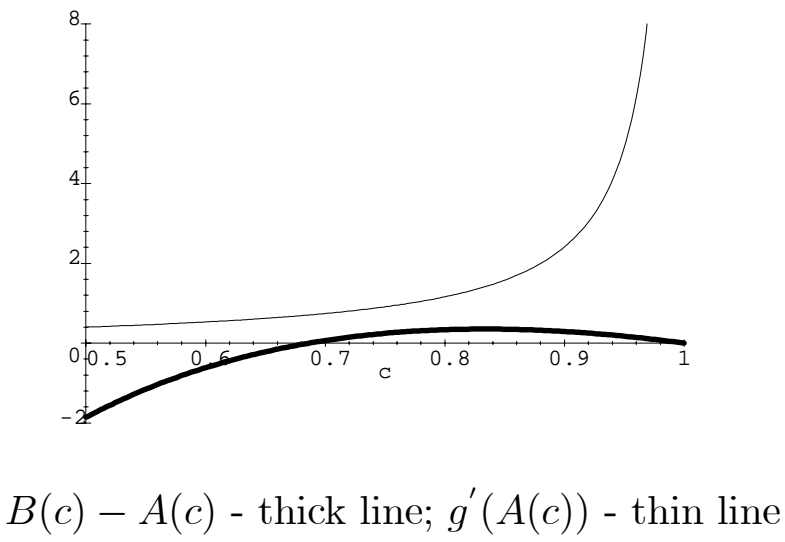

Numerical calculations reveal that $\alpha^{*}=\frac{1}{e}$ is (an approximate) solution to the equation $R^{\prime}(\alpha)=0$. Hence the optimal prize structure is $V_{1}=1-\frac{1}{e} \approx 0.63$, and $V_{2}=\frac{1}{e} \approx 0.37$. The ratio of prizes is $\frac{V_{1}}{V_{2}}=e-1 \approx 1$. 71, and the difference $V_{1}-V_{2}$ is about one quarter of the prize sum.

\section{Concluding Comments}

We have studied the optimal prize structure in multi-prize contests where players have private information about their abilities. In order to maximize the expected sum of bids, the designer should award a single prize if contestants have linear or concave cost functions. If the contestants have convex cost functions, then two prizes (or more) may be optimal. The optimal proportion between the prizes' values depends then on the number of contestants, the distribution of abilities in the population, and on the exact form of the cost function.

What can we say about Galton's proposal to have a first prize which is three times higher than the second prize ? Assume that contestants have linear cost functions. It can be easily shown that, when the number of contestants goes to infinity (this was the actual case envisaged by Galton), the average difference between the marginal effects of the two prizes goes to zero. Hence, the designer becomes indifferent between awarding one prize or two prizes (in any proportion). In particular, Galton's $3: 1$ proportion becomes approximately optimal.

We see several avenues for future research: 1) Combine the model of private information about abilities with the earlier models where output depend stochastically on effort, and only output can be observed. 2) Perform comparative static exercises about the fine effects of changes in the distribution of abilities or in the 
form of the cost functions when these are convex. 3) Compare simultaneous contests with sequential contests. 4) Conduct empirical studies about the optimal prize structure in observed contests, such as architectural competitions.

Francis Galton concluded his article with the following remark:

"I now commend the subject to mathematicians in the belief that those who are capable, which I am not, of treating it more thoroughly, may find that further investigations will repay trouble in unexpected directions" (Galton, 1902)

The challenge was immediately picked by the famous statistician Karl Pearson, at that time editor of Biometrika. His notes at the end of Galton's article contain a complete solution of the statistical problem posed (calculate the ratio of expected differences among order statistics), but does not refer to the original question of prize allocation in contests. It is now up to the reader to decide whether we, humble economists, have made a contribution.

\section{References}

Amann, E., and Leininger, W. (1996)." Asymmetric all-pay Auctions with Incomplete Information: The Two-Player Case". Games and Economic Behavior, 14, $1-18$.

Baye, M., Kovenock, D., and de Vries, C. (1996)."The All-Pay Auction with Complete Information", Economic Theory, 8 , 291-305.

Barut, Y., and Kovenock, D. (1998)." The Symmetric Multiple Prize All-Pay Auction with Complete Information" , European Journal of Political Economy, 14, 627-644.

Clark, D., and Riis, C. (1998)."Competition over More than One Prize.", American Economic Review, 88, 276-289.

Galton, F. (1902). "The Most Suitable Proportion Between The Values Of First And Second Prizes", Biometrika, 1(4), 385-390

Glazer, A., and Hassin, R. (1988). "Optimal Contests", Economic Inquiry 26, 133-143.

Green, J., and Stokey, N. (1983)." A Comparison of Tournaments and Contracts." Journal of Political Economy , 91(3), 349-364.

Hilman, A., and Riley, J. (1989)."Politically Contestable Rents and Transfers". Economics and Politics, 1, 17-39.

Hilman, A., and Samet, D. (1987)." Dissipation of Contestable Rents by Small Numbers of Contenders". , Public Choice, 54, 63-82. 
Krishna, V., and Morgan, J. (1997)." An Analysis of the War of Attrition and the All-Pay Auction." , Journal of Economic Theory, 72, 343-362.

Lazear, E., and Rosen, S.(1981)."Rank Order Tournaments as Optimum Labor Contracts" , Journal of Political Economy, 89, 841-864.

Nalebuff, B., and Stiglitz, J. (1983). "Prizes and Incentives: Towards a General Theory of Compensation and competition.", Bell Journal of Economics, 14, 2143.

Pearson, K. (1902). "Note On Francis Galton's Problem", Biometrika, 1(4), 390-399.

Rosen, S. (1986)."Prizes and Incentive in Elimination Tournaments", American Economic Review, 76, 701-715.

Tullock, G. (1980), "Efficient rent-seeking", in J.Buchanan et.al., eds, Towards a Theory of the Rent-Seeking Society, Texas A\&M University Press, College Station.

Varian, H. (1980), "A Model of Sales", American Economic Review, 651-659

Weber, R. (1985), "Auctions and competitive bidding", in H.P. Young, ed., Fair Allocation, American Mathematical Society, 143-170

\section{Appendix}

Proof of Proposition 3.1: Assume that all contestants in the set $K \backslash\{i\}$ bid according to $b(\cdot)$, and assume that the bid function is strictly monotonic and differentiable.

Player $i$ 's maximization problem reads:

$$
\max _{x}\left[V_{1}\left(1-F\left(b^{-1}(x)\right)\right)^{k-1}+(k-1) V_{2} F\left(b^{-1}(x)\right)\left(1-F\left(b^{-1}(x)\right)\right)^{k-2}-c x\right]
$$

Let $y(\cdot)$ denote the inverse function of $b(\cdot)$. Using strict monotonicity and symmetry, the first order condition is:

$$
\begin{aligned}
1= & -(k-1)\left(V_{1}-V_{2}\right) y^{\prime} \frac{1}{y}(1-F(y))^{k-2} F^{\prime}(y)- \\
& (k-1)(k-2) V_{2} y^{\prime} \frac{1}{y} F(y)(1-F(y))^{k-3} F^{\prime}(y)
\end{aligned}
$$

Note that the right hand side of the FOC is a function of $y$ only (i.e., this is a differential equation with separated variables).

A contestant with the lowest possible ability $c=1$ can either never win a prize (if $k>2$ ) or wins for sure the second prize (if $k=2$ ). Hence the optimal bid of this type is always zero, and this yields the boundary condition $y(0)=1$, 
The solution to the differential equation with the boundary condition is given by :

$$
\begin{aligned}
\int_{x}^{0} d t= & -V_{1}((k-1)) \int_{y}^{1} \frac{1}{t}(1-F(t))^{k-2} F^{\prime}(t) d t+ \\
& -V_{2}(k-1) \int_{y}^{1} \frac{1}{t}(1-F(t))^{k-3}[1-(k-1) F(t)] F^{\prime}(t) d t
\end{aligned}
$$

Denote

$$
\begin{aligned}
G(y)= & V_{1}((k-1)) \int_{y}^{1} \frac{1}{t}(1-F(t))^{k-2} F^{\prime}(t) d t+ \\
& V_{2}(k-1) \int_{y}^{1} \frac{1}{t}(1-F(t))^{k-3}[1-(k-1) F(t)] F^{\prime}(t) d t
\end{aligned}
$$

We obtain that $x=G(y)=G\left(b^{-1}(x)\right)$, and therefore that $b(\cdot)=G(\cdot)$. Thus, the bid function of every player is given by $b(c)=A(c) V_{1}+B(c) V_{2}$, where:

$$
\begin{gathered}
A(c)=(k-1) \int_{c}^{1} \frac{1}{a}(1-F(a))^{k-2} F^{\prime}(a) d a \\
B(c)=(k-1) \int_{c}^{1} \frac{1}{a}(1-F(a))^{k-3}[(k-1) F(a)-1] F^{\prime}(a) d a
\end{gathered}
$$

We now check that the candidate equilibrium function $b(\cdot)$ is strictly monotonic decreasing (it is clearly differentiable). Note first that

$$
A^{\prime}(c)=-(k-1) \frac{1}{c}(1-F(c))^{k-2} F^{\prime}(c)<0
$$

for all $c \in[m, 1)$. We have also

$$
B^{\prime}(c)=(k-1) \frac{1}{c}(1-F(c))^{k-3} F^{\prime}(c)\left[(1-(k-1) F(c)] F^{\prime}(c)\right.
$$

Because $V_{1} \geq V_{2}$ we obtain for all $c \in[m, 1)$ :

$$
\begin{aligned}
b^{\prime}(c) & =A^{\prime}(c) V_{1}+B^{\prime}(c) V_{2} \\
& \leq V_{2}\left(A^{\prime}(c)+B^{\prime}(c)\right) \\
& \left.=-V_{2}(k-1)(k-2) \frac{1}{c} F(c)(1-F(c))^{k-3} F^{\prime}(c)\right) \\
& <0
\end{aligned}
$$


Assuming that all contestants other than $i$ bid according to $b(\cdot)$, we finally need to show that, for any type $c$ of player $i$, the bid $b(c)$ maximizes the expected utility of that type. The necessary first-order condition is clearly satisfied (since this is how we "guessed" $b(c)$ to start with). We now show that a sufficient second-order condition (called "pseudoconcavity") is satisfied. Let

$$
\pi(x, c)=V_{1}\left(1-F\left(b^{-1}(x)\right)^{k-1}+(k-1) V_{2} F\left(b^{-1}(x)\right)\left(1-F\left(b^{-1}(x)\right)^{k-2}-c x\right. \text { be }\right.
$$

the expected utility of player $i$ with type $c$ that makes a bid $x$.

We will show that the derivative $\pi_{x}(c, x)$ is nonnegative if $x$ is smaller than $b(c)$ and nonpositive if $x$ is larger than $b(c)$. As $\pi(x, c)$ is continuous in $x$, this implies that $\pi(x, c)$ is maximized at $x=b(c)$.

Note that $\pi_{x}(x, c)=-(k-1)\left(V_{1}-V_{2}\right) \frac{d b^{-1}(x)}{d x}\left(1-F\left(b^{-1}(x)\right)\right)^{k-2} F^{\prime}\left(b^{-1}(x)\right)-$

$(k-1)(k-2) V_{2} \frac{d b^{-1}(x)}{d x} F\left(b^{-1}(x)\right)\left(1-F\left(b^{-1}(x)\right)\right)^{k-3} F^{\prime}\left(b^{-1}(x)\right)-c$.

Let $x<b(c)$, and let $\widehat{c}$ be the type who is supposed to bid $x$, that is $b(\widehat{c})=x$. Note that $\widehat{c}>c$ since $b(\cdot)$ is strictly decreasing.

Differentiating $\pi_{x}(x, c)$ with respect to $c$ yields $\pi_{x c}(x, c)=-1<0$. That is, the function $\pi_{x}(x, \cdot)$ is decreasing in $c$. Since $\widehat{c}>c$, we obtain $\pi_{x}(x, c) \geq \pi_{x}(x, \widehat{c})$

Since $x=b(\widehat{c})$ we obtain by the first order condition that $\pi_{x}(x, \widehat{c})=0$,and therefore that $\pi_{x}(x, c) \geq 0$ for every $x<b(c)$.

A similar argument shows that $\pi_{x}(x, c) \leq 0$ for every $x>b(c)$.

\section{The symmetric equilibrium with $p$ prizes:}

Fix agent $i$, and let $F_{s}(a), 1 \leq s \leq p$, denote the probability that agent $i$ with type $a$ meets $k-1$ competitors such that $s-1$ of them have lower types, and $k-s$ have higher types. Recall that in equilibrium we expect $i$ to bid more than competitors with higher types (lower ability). Hence $F_{s}(\cdot)$ is exactly the probability of winning the $s^{\prime}$ th prize. We have then

$$
F_{s}(a)=\frac{(k-1) !}{(s-1) !(k-s) !}(1-F(a))^{k-s}(F(a))^{s-1}
$$

The corresponding derivatives are given by

$$
F_{1}^{\prime}(a)=-(k-1)(1-F(a))^{k-2}
$$

and by

$$
\begin{aligned}
F_{s}^{\prime}(a)= & \frac{(k-1) !}{(s-1) !(k-s) !}(1-F(a))^{k-s-1}(F(a))^{s-2} F^{\prime}(a) \\
& \cdot[(1-k) F(a)+(s-1)]
\end{aligned}
$$

for $s>1$. 
Note that $A(c)=\int_{c}^{1}-\frac{1}{a} F_{1}^{\prime}(a) d a$ and that $B(c)=\int_{c}^{1}-\frac{1}{a} F_{2}^{\prime}(a) d a$. Analogously to the case of two prizes, the equilibrium bid for any number of prizes $p$, any number of contestants $k \geq p$ with linear cost functions is given by:

$$
b(c)=\sum_{s=1}^{p} V_{s} \cdot \int_{c}^{1}-\frac{1}{a} F_{s}^{\prime}(a) d a
$$

Proof of Proposition 3.2: Recall that: $A(c)=(k-1) \int_{c}^{1} \frac{1}{a}(1-F(a))^{k-2} F^{\prime}(a) d a$ and $B(c)=(k-1) \int_{c}^{1} \frac{1}{a}(1-F(a))^{k-3}[(k-1) F(a)-1] F^{\prime}(a) d a$

1. This is obvious by definition.

2. $A(c)>0$ for $c \in[m, 1)$ is obvious by definition. Further we have $A^{\prime}(c)=$ $-(k-1) \frac{1}{c}(1-F(c))^{k-2} F^{\prime}(c)<0$ for all $c \in[m, 1)$ and $A^{\prime}(1)=0$.

3. $B^{\prime}(c)=(k-1) \frac{1}{c}(1-F(c))^{k-3} F^{\prime}(c)[(1-(k-1) F(c)]$

For $c^{*}$ such that $F\left(c^{*}\right)=\frac{1}{k-1}$ we obtain $B^{\prime}\left(c^{*}\right)=0$. Moreover, $B^{\prime}(c)>0$ for all $c \in\left[m, c^{*}\right)$, and $B^{\prime}(c)<0$ for all $c \in\left(c^{*}, 1\right)$. Finally, $B^{\prime}(1)=0$.

4. For for all $c \in\left[c^{*}, 1\right)$ we obtain that

$$
\begin{aligned}
& B^{\prime}(c)|-| A^{\prime}(c) \mid \\
= & -B^{\prime}(c)+A^{\prime}(c) \\
= & (k-1) \frac{1}{c}(1-F(c))^{k-3} F^{\prime}(c)(k F(c)-2)
\end{aligned}
$$

For $k>2$ we obtain that $\left|B^{\prime}(c)\right|-\left|A^{\prime}(c)\right|$ is positive for $c$ close enough to 1 (since $k F(c)>2$ for such types.)

5. We have

$$
\begin{aligned}
B(m)= & (k-1)\left(\int_{m}^{c^{*}} \frac{1}{a}(1-F(a))^{k-3}[(k-1) F(a)-1] F^{\prime}(a) d a\right. \\
& +(k-1)\left(\int_{c^{*}}^{1} \frac{1}{a}(1-F(a))^{k-3}[(k-1) F(a)-1] F^{\prime}(a) d a\right. \\
< & (k-1) \int_{m}^{1}(1-F(a))^{k-3}[(k-1) F(a)-1] F^{\prime}(a) d a
\end{aligned}
$$

The last inequality follows by noting that the integrand in the first integral is negative and that the integrand of the second integral is positive. If we multiply both integrands by the increasing function $h(a)=a$ we strictly increase the value of the sum of the two integrals. In order to prove that 
$B(m)<0$ it is then enough to prove that $\int_{m}^{1}(1-F(a))^{k-3}[(k-1) F(a)-$ 1] $F^{\prime}(a) d a=0$. By the change of variable $z=F(a)$,we obtain

$$
\begin{aligned}
& \int_{m}^{1}(1-F(a))^{k-3}[(k-1) F(a)-1] F^{\prime}(a) d a \\
= & \int_{0}^{1}(1-z)^{k-3}[(k-1) z-1] d z=0
\end{aligned}
$$

6. This follows by combining all properties above.

7. We know that $B(c)-A(c)>0$ for all $c \in\left[m, c^{* *}\right)$ and that $B(c)-A(c)<0$ for all $c \in\left(c^{* *}, 1\right)$. This yields:

$$
\begin{aligned}
& \int_{m}^{1}(B(c)-A(c)) F^{\prime}(c) d c \\
= & \int_{m}^{c^{* *}}(B(c)-A(c)) F^{\prime}(c) d c+\int_{c^{* *}}^{1}(B(c)-A(c)) F^{\prime}(c) d c \\
= & (k-1) \int_{m}^{c^{* *}}\left[\int_{c}^{1} \frac{(1-F(a))^{k-3}}{a}(k F(a)-2) F^{\prime}(a) d a\right] F^{\prime}(c) d c+ \\
& (k-1) \int_{c^{* *}}^{1}\left[\int_{c}^{1} \frac{(1-F(a))^{k-3}}{a}(k F(a)-2) F^{\prime}(a) d a\right] F^{\prime}(c) d c \\
< & (k-1) \frac{1}{c^{* *}} \int_{m}^{c^{* *}}\left[\int_{c}^{1}(1-F(a))^{k-3}(k F(a)-2) F^{\prime}(a) d a\right] F^{\prime}(c) d c+ \\
& (k-1) \frac{1}{c^{* *}} \int_{c^{* *}}^{1}\left[\int_{c}^{1}(1-F(a))^{k-3}(k F(a)-2) F^{\prime}(a) d a\right] F^{\prime}(c) d c \\
= & (k-1) \frac{1}{c^{* *}} \int_{m}^{1}\left[\int_{c}^{1}(1-F(a))^{k-3}(k F(a)-2) F^{\prime}(a) d a\right] F^{\prime}(c) d c \\
= & \frac{1}{c^{* *}} \int_{0}^{1}\left[\int_{v}^{1}(k-1)(1-z)^{k-3}(k z-2) d z\right] d v=0
\end{aligned}
$$

The last equality follows by the changes of variables $F(a)=z$ and $F(c)=v$

Proof of Proposition 4.1: Assume that all contestants in the set $K \backslash\{i\}$ bid according to $b(\cdot)$, and assume that the bid function is strictly monotonic and differentiable. Let $y(\cdot)$ denote the inverse function of $b(\cdot)$.

Player $i$ 's maximization problem reads:

$$
\max _{x}\left[V_{1}\left(1-F\left(b^{-1}(x)\right)\right)^{k-1}+(k-1) V_{2} F\left(b^{-1}(x)\right)\left(1-F\left(b^{-1}(x)\right)\right)^{k-2}-c \gamma(x)\right]
$$

Using strict monotonicity and symmetry, the first order condition is: 


$$
\begin{aligned}
\gamma^{\prime}(x)= & -(k-1)\left(V_{1}-V_{2}\right) y^{\prime} \frac{1}{y}(1-F(y))^{k-2} F^{\prime}(y)- \\
& (k-1)(k-2) V_{2} y^{\prime} \frac{1}{y} F(y)(1-F(y))^{k-3} F^{\prime}(y)
\end{aligned}
$$

Note that this is also an ordinary differential equation with separated variables (i.e. the left hand side of the first equation is a function of $x$ only, while the right hand side is a function of $y$ only.

Integration and the use of the boundary condition $y(1)=0$ yield $\gamma(x)=G(y)$, where $G(y)$ is defined exactly as in the proof of Proposition 3.1 (see equation 7.2) Hence, we obtain that $x=\gamma^{-1}(G(y))=g\left(G\left(b^{-1}(x)\right)\right)$ and that $b(\cdot)=g(G(\cdot))$.

Note that the candidate equilibrium bid function $b(c)=\gamma^{-1}\left(A(c) V_{1}+B(c) V_{2}\right)=$ $g\left(A(c) V_{1}+B(c) V_{2}\right)$ is strictly decreasing since, for all $c \in[m, 1)$, it holds:

$$
\begin{aligned}
& \frac{d g}{d c}\left(A(c) V_{1}+B(c) V_{2}\right) \\
= & g^{\prime}\left(A(c) V_{1}+B(c) V_{2}\right) \cdot\left(A^{\prime}(c) V_{1}+B^{\prime}(c) V_{2}\right)<0
\end{aligned}
$$

The last inequality follows because $g^{\prime}(\cdot)>0$ by assumption, while $A^{\prime}(c) V_{1}+$ $B^{\prime}(c) V_{2}<0$ since this is the derivative of the bid function with linear cost functions (see proof of Proposition 3.1).

For the sufficient second-order condition we proceed exactly as in the proof of Proposition 3.1. Using the notation employed in that proof, we have

and

$$
\pi(x, c)=V_{1}\left(1-F\left(b^{-1}(x)\right)^{k-1}+(k-1) V_{2} F\left(b^{-1}(x)\right)\left(1-F\left(b^{-1}(x)\right)^{k-2}-c \gamma(x)\right.\right.
$$

$\pi_{x}(x, c)=-(k-1)\left(V_{1}-V_{2}\right) \frac{d b^{-1}(x)}{d x}\left(1-F\left(b^{-1}(x)\right)\right)^{k-2} F^{\prime}\left(b^{-1}(x)\right)-$

$(k-1)(k-2) V_{2} \frac{d b^{-1}(x)}{d x} F\left(b^{-1}(x)\right)\left(1-F\left(b^{-1}(x)\right)\right)^{k-3} F^{\prime}\left(b^{-1}(x)\right)-c \gamma^{\prime}(x)$.

Differentiating $\pi_{x}(x, c)$ with respect to $c$ yields $\pi_{x c}(x, c)=-\gamma^{\prime}(x)<0$. That is, the function $\pi_{x}(x, \cdot)$ is decreasing in $c$, exactly as in the linear case. The proof of pseudoconcavity is concluded as in that case.

Analogously to the case of two prizes, the equilibrium bid for any number of prizes $p$, and any for number of contestants $k \geq p$ with cost functions of the form $c \gamma(x)$ is given by:

$$
b(c)=\gamma^{-1}\left(\sum_{s=1}^{p} V_{s} \cdot \int_{c}^{1}-\frac{1}{a} F_{s}^{\prime}(a) d a\right)
$$

where $F_{s}^{\prime}(a)$ is given in formulas 7.3 and 7.4. 